\title{
CASPER: Congestion Aware Selection of Path with Efficient Routing in Multimedia Networks
}

\author{
Mohammad S. Obaidat ${ }^{\star}$, Sanjay K. Dhurandher** and Khushboo Diwakar**
}

\begin{abstract}
In earlier days, most of the data carried on communication networks was textual data requiring limited bandwidth. With the rise of multimedia and network technologies, the bandwidth requirements of data have increased considerably. If a network link at any time is not able to meet the minimum bandwidth requirement of data, data transmission at that path becomes difficult, which leads to network congestion. This causes delay in data transmission and might also lead to packet drops in the network. The retransmission of these lost packets would aggravate the situation and jam the network. In this paper, we aim at providing a solution to the problem of network congestion in mobile ad hoc networks [1,2] by designing a protocol that performs routing intelligently and minimizes the delay in data transmission. Our Objective is to move the traffic away from the shortest path obtained by a suitable shortest path calculation algorithm to a less congested path so as to minimize the number of packet drops during data transmission and to avoid unnecessary delay. For this we have proposed a protocol named as Congestion Aware Selection Of Path With Efficient Routing (CASPER). Here, a router runs the shortest path algorithm after pruning those links that violate a given set of constraints. The proposed protocol has been compared with two link state protocols namely, OSPF $[3,4]$ and $\operatorname{OLSR}[5,6,7,8]$.The results achieved show that our protocol performs better in terms of network throughput and transmission delay in case of bulky data transmission.
\end{abstract}

Keywords-Routing, Multimedia Networks, Congestion-aware Selection, MANET, CASPER, Performance Evaluation

\section{INTRODUCTION}

Mobile Ad Hoc Networks (MANETs) [1,2] have the property of unstable link connectivity between nodes that keeps on breaking and then reforming due to the mobility of the nodes. As the links are highly unstable, the link bandwidth too keeps on changing. Due to this feature of MANETs, transmission of bulky data with high bandwidth requirements might become a problem. The protocol proposed in this work aims at eradicating this problem. This protocol is a link state protocol that maintains the link state of all the nodes in the network.

\subsection{Motivation and Contributions}

The use of a congestion reduction technique is important while transmitting bulky data in

\footnotetext{
Manuscript received April 13, 2011; accepted May 15, 2011.

Corresponding Author: Mohammad S. Obaidat

* Department of CS \& SE, Monmouth University, NJ, USA (obaidat@monmouth.edu)

** CAITFS, Division of Information Technology, Netaji Subhas Institute of Technology, University of Delhi, New Delhi, India (dhurandher@rediffmail.com,diwakar_khushboo@yahoo.co.in)
} 
MANETs. Due to the different bandwidths of links between nodes in MANETs, the shortest path in terms of number of hop counts between two nodes may not always be suitable to transmit bulky data having high bandwidth requirement. Moreover, not all the links between the nodes in a network may satisfy the minimum bandwidth requirement of the data. This causes unnecessary delay and jitter and also drops the packet delivery ratio considerably. In order to cure this problem, we have devised a new protocol CASPER that applies congestion reduction technique while transmitting data packets. It calculates constrained shortest path between nodes in a circular restricted topology. Application of the Constrained Shortest Path First (CSPF) algorithm in OSPF has already been suggested in [9]. Our Protocol also helps in reducing the number of control packets as lesser number of nodes are involved in the shortest path calculation. This further helps in the routing process due to the inclusion of only those nodes that lie in the restricted circular topology. It lessens the probability of congestion in the network as only those links are processed, which satisfy the minimum bandwidth criteria depending upon the bandwidth of the message to be transmitted. It also leads to an efficient usage of the routing table space as only those routes are stored, which are obtained through constrained circular routing. Our protocol uses OSPF $[3,4]$ as the base protocol. OSPF $[3,4]$ is a very popular link state routing protocol which calculates path between nodes using a suitable shortest path algorithm. Various adjacency reduction techniques $[20,21]$ and traffic engineering $[22,23]$ have already been implemented in OSPF, however, our algorithm provides yet another way to achieve congestion reduction in MANETs. Also there are protocols that target energy saving that have been reported such as the DEESR scheme [24].

The paper has been divided into six sections. Section 2 describes the existing link state protocols used in MANETS. Sections 3 and 4 present the algorithm and the pseudo code of the proposed protocol, respectively. Section 5 presents the simulation metrics and the analysis of the proposed protocol CASPER based on the results of the simulation. Finally, Section 6 presents the conclusion and the future work that can be carried out of this work. Sections 6,7 and 8 present the conclusion, future work and references, respectively.

\section{Benchmark Protocols Used}

As the proposed protocol is a link state protocol, it has been compared with OSPF and OLSR which are the other link state protocols used in MANETs. The following sub-sections present a brief overview of these routing protocols. Section 2.1 explains the OSPF protocol $[3,4]$ which is a link state interior gateway protocol used in MANETS. Section 2.3 contains explanation for the OLSR protocol $[5,6,7,8]$. Our protocol uses OSPF as the base protocol and adds-on the constrained circular routing technique to it. Section 2.3 explains the Constrained Shortest path calculation algorithm that is not new to our research.

\subsection{Open Shortest Path First (OSPF)}

OSPF $[3,4]$ is an interior gateway protocol that maintains link state database for efficient routing between nodes. It floods the routing information to obtain a complete overview of the network topology. The data exchanges that take place during this process are called Link State Advertisements (LSA) [3, 4]. Each router stores these LSA's in its Link State Database (LSDB). OSPF uses a shortest path algorithm to find and store the shortest path from one node to another. 
Dijkstra's algorithm determines the least cost path from a router to other nodes. A periodic broadcast of the updates about the state of its links is done by each router to synchronize its LSDB. All links between nodes are assigned a cost. It selects the best route by finding the minimum cost path from the source to the destination. Each node also maintains a routing table to store paths to other nodes in the network.

OSPF keeps track of the state of all the links between various nodes in a network topology, which makes it a Link State Protocol. Routers that share a link become neighbors and each neighbor is elected via exchange of Hello packets. These Hello packets are multicast periodically and are used to determine if the neighbor is up. A router becomes neighbor of another router if it sees itself mentioned in the routers Hello packet. This guarantees a two way communication between routers.

After forming neighbors, the next step is the formation of Adjacencies routers. Adjacency routers are routers that connect to a common network. They are responsible for database exchange and synchronization of their LSDBs.

For reducing the amount of information exchange on a particular segment, OSPF performs election of a Designated Router and a Backup Designated Router, on each multi access segment. This Designated Router acts as a central point for information exchange as every router exchanges information with a Designated Router instead of exchanging with every other node in the segment. This information is then replayed to every other router by the Designated Router. A Backup Designated Router is elected to replace the Designated Router incase it goes down. The election of Designated Router and Backup Designated Router is done through an election algorithm. In our protocol, we have taken the whole network area to be a single autonomous system.

\subsection{Optimized Link State Routing}

The Optimized Link State Routing Protocol (OLSR) is a MANET protocol that operates as a table driven, proactive protocol, i.e., it exchanges topology information with other nodes of the network regularly. It uses Hello messages to discover link state information between nodes in a network and uses Topology Control (TC) messages $[5,6,7,8]$ to distribute this information throughout the mobile ad-hoc network. Each node uses this topology information to compute the next hop destinations to other nodes.

A set of neighbors of each node is selected as "multipoint relays" (MPR) [10,11] by the node for forwarding control traffic. The MPR's are selected by each node such that, there exists a path to each of its neighbor via MPR. Multipoint relays (MPRs) are selected by a node from amongst its one hop neighbors. These MPR nodes are used to forward TC messages for spreading link state information in the network. MPR has accessibility to the nodes which selected it as MPR. The route calculation procedure requires the MPR nodes to form route from a source node to destination.

OLSR does not employ a Designated Router like OSPF. Rather it has MPRs which facilitate efficient routing and minimize the overhead of flooding messages in the network through reduction of unnecessary transmissions in an area. Each node maintains information about the set of neighbors that has selected as MPR through the use of Hello Messages.

\subsection{Constrained Shortest Path First}

This algorithm is not new to our paper and has also been used in [9]. CSPF [9] algorithm cal- 
culates the shortest path between the nodes after discarding those links that violate a given set of constraints. These constraints can be: minimum bandwidth required per link, end-to-end delay, maximum number of links traversed, etc. The constraint used in our protocol is minimum bandwidth requirement per link. The concept of Constrained Shortest Path First has been explained with the help of a diagram.

Consider this network where a route has to be computed from router-1 to the router-6.The route should satisfy the bandwidth constraint of 60- units, and link cost for each link is based on hop-count. Based on this requirement CSPF will give a path of $1 \rightarrow 4 \rightarrow 5 \rightarrow 6$ as it satisfies the minimum bandwidth requirement of the message. A simple OSPF would select either the path $1 \rightarrow 2 \rightarrow 3 \rightarrow 6$ or $1 \rightarrow 4 \rightarrow 5 \rightarrow 6$ with equal probability. However, the path 1 $\rightarrow 2 \rightarrow 3 \rightarrow 4$ will cause congestion in this case and should be avoided as the link between node 1 and 2 does not satisfy the minimum bandwidth requirement of the message.

OLSR $[5,6,7,8]$ and OSPF $[3,4]$ do not provide any provisions for sensing the link quality. As will be seen later, on the other hand, CASPER proves to be a better protocol than OSPF and OLSR while dealing with bulky messages. This is due to the congestion reduction technique used in CASPER, which is not present in the other two protocols. CASPER employs Constrained Shortest path calculation between the source and the destination nodes in a circular restricted topology.

\section{Proposed Protocol}

In this paper, we have proposed a circle based routing technique for forwarding data. Instead of calculating and storing the path from the source node to all the nodes in the topology, we calculate and store the path from the source node to the destination node having only those intermediate nodes that lie in a restricted circular topology created by our algorithm. Also the shortest path is calculated such that it satisfies the minimum bandwidth requirement of the data to be sent so as to minimize the chances of congestion in the network. Thus, instead of sending the complete topology graph for shortest path calculation, we send a portion of the graph extracted using constrained circular routing technique. This technique helps in reducing the number of control packets as lesser number of nodes are involved in the shortest path calculation and which is so in the routing process as well. This reduces the probability of congestion in the network. This is due to the fact, that, this is done in order to satisfy the minimum bandwidth criteria taking into consideration the bandwidth requirement of the message that needs to be transmitted. This further helps in better routing table space since only those routes that are obtained through the constrained circular routing are stored in the routing table. This is explained through Figure 1 , where the number mentioned in units for a link between two nodes represents the bandwidth of the link.

Suppose the source node, Node 1 wants to send data to Node 6. Node 1 first obtains the location information of the destination and its neighbor nodes. It then finds the midpoint of the straight line distance between itself and Node 6.The distance between the midpoint and Node 1 is then used as the radius to restrict the topology circularly and we consider only those nodes that lie inside this circle for Constrained Shortest Path calculation.

In this example Node 2 and Node 5 lie inside the circle and so are used for path calculation. Let us say that the bandwidth required for the message to be sent by Node 1 is 60 units. It is seen that the current topology does not satisfy the minimum bandwidth requirement of the message 


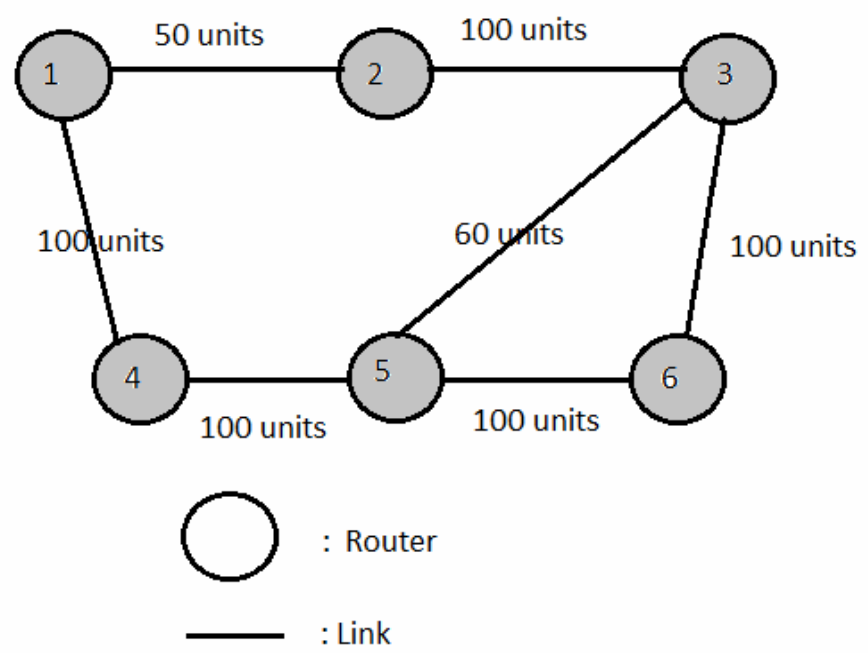

Fig. 1: Constrained Shortest Path First (CSPF) [17]
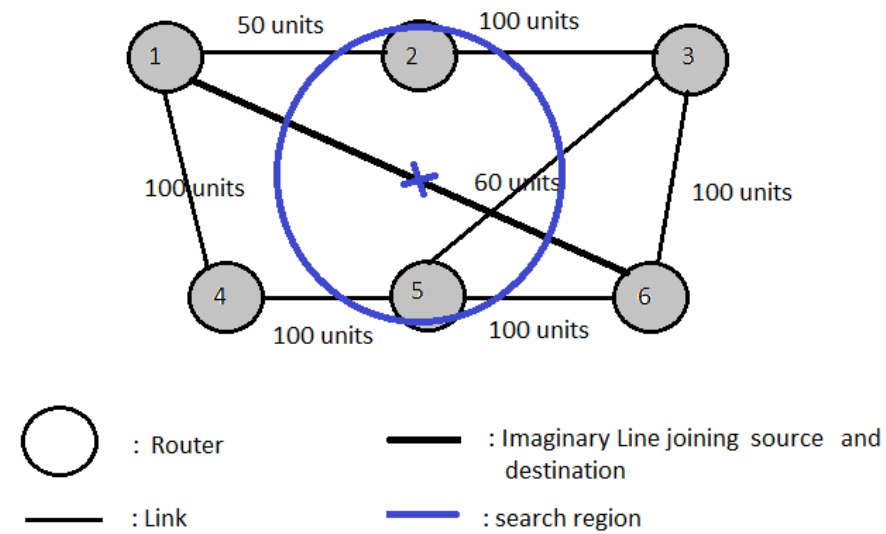

Fig. 2. Illustration of the Constrained Circular Routing

and may cause congestion, hence, the radius of the topology is increased by the initial value of the radius and the constrained shortest path calculation is carried out. In case no route is found after incrementing the radius, the radius is again incremented and CSPF calculation is done. The radius is always increased by an amount equal to the distance between the source node and the midpoint. In case no path is found after incrementing the radius, the Constrained Shortest Path calculation is carried out on the complete topology and a path is tried to be found.

Section 3.1 deals with assumptions related to the algorithm. Section 3.2 explains the basic procedure of routing used in CASPER.

\subsection{Assumptions}

The various assumptions made while designing the algorithm are as below:

- The node is capable of extracting location coordinates of itself by querying its GPS device [12]. 
- A node can obtain the location information of the destination node as well as the neighbor nodes using a suitable location updating algorithm.

- Nodes have moderate speed and all nodes fully participate in forwarding data to other nodes. The Network Topology does not change frequently.

\subsection{Topology Restriction Algorithm}

- Find Source and Destination Coordinates.

- Find midpoint of the straight line joining Source node and Destination node.

- With midpoint as center and radius (calculated using Radius Calculation Algorithm) draw a circle and include Source node, Destination node and other nodes within the circle in the final topology.

\subsection{Radius Calculation Algorithm}

- Take one half of the distance between midpoint (calculated in the Topology Restriction Algorithm) and Source node as Radius (First Radius) in $1^{\text {st }}$ iteration and find route between Source and Destination Node after applying Topology Restriction Algorithm.

- If no route between Source and Destination Node is found in $1^{\text {st }}$ iteration update Radius as Radius $=$ Radius + First Radius .

- If no route between Source and Destination Node is found in $2^{\text {nd }}$ iteration, then update Radius as Radius $=$ Radius + First Radius.

- Else, if no route is found in $3^{\text {rd }}$ iteration also, consider Whole Topology for path calculation.

\subsection{Constrained Shortest Path First Algorithm}

This algorithm is not original to our research and has been explained in section 2. Here each link between nodes in the topology is compared with the message bandwidth to prune out those links that do not satisfy the minimum bandwidth criteria and may lead to congestion in network. Only those links that have bandwidth greater or equal than message are chosen for shortest path calculation.

\subsection{Basic procedure for Data Forwarding}

- If route table exists, the source host consults the table, looks up the route to destination node, and forwards the packet.

- Otherwise, it invokes the procedure Changed Route Discovery (), explained in Section 3.6.

- If no node is found within the range to forward the data packet, then the procedure Original_Route Discovery () is invoked.

\subsection{Changed_Route_Discovery()}

Here the Topology Restriction, Radius Calculation and CSPF methods are invoked.

- Source and Destination Coordinates are extracted.

- The Midpoint of the straight line joining the Source and Destination Node is calculated.

- With Midpoint as center and Radius calculated using Radius Calculation Algorithm an imaginary circle is drawn, only the topology inside the circle and Source and Destination 
Nodes is considered.

- Route calculation from source to destination node is done using Constrained Shortest Path First Algorithm.

- If no route exists, Radius is doubled and Topology restriction method () is invoked again and route is calculated; else Data Packet is forwarded to Destination.

- If no Route exists on increasing Topology, then Radius = Radius + First Radius and Topology restriction method () is invoked again and route is calculated; else Data Packet is forwarded to Destination.

- Else; if no route found within this topology Original_Route_Discovery() is invoked.

\subsection{Original_Route_Discovery()}

- The Whole Topology containing all the available nodes is used for Constrained Shortest Path First Calculation between all the nodes.

- The Route between Source and Destination Node is looked up in the routing table.

- If route exists, Data Packet is forwarded.

- Else; Packet is dropped as no route exists.

The flowchart in Figure 3 shows how Data Forwarding is performed. If there is a message which needs to be transmitted, the route is discovered for it. Every node which lies in the circle drawn with radius $\mathrm{R}$ (calculated using Radius Calculation Algorithm) is considered for route

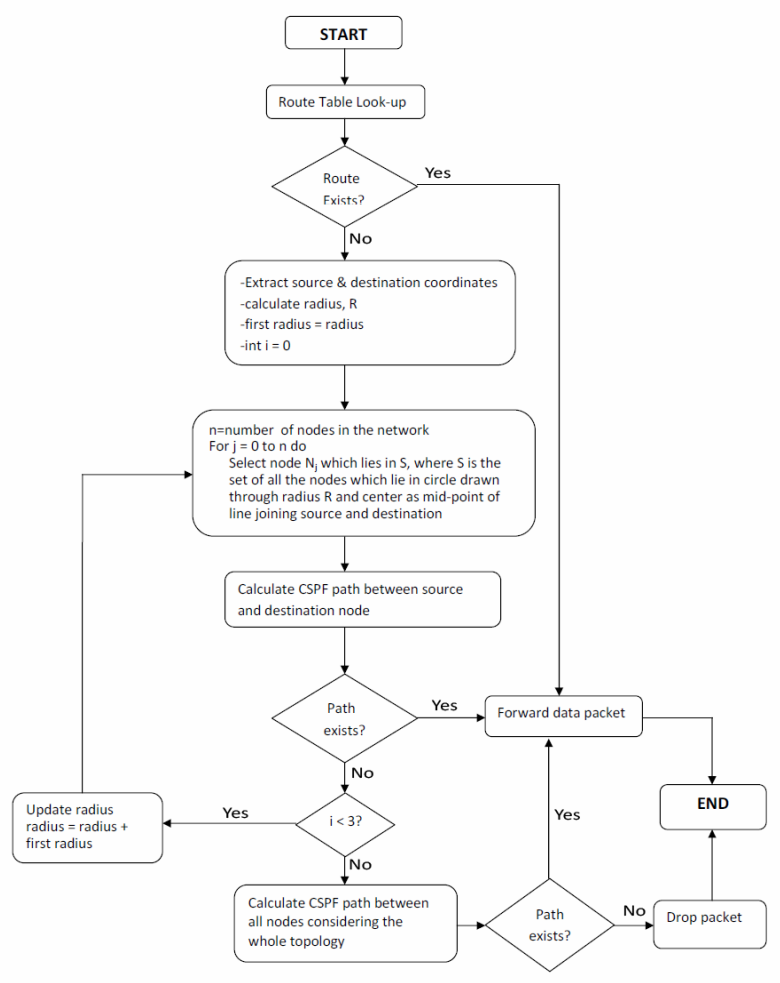

Fig. 3. Flowchart Depicting Data Transfer between Nodes 
selection. Each time when the protocol fails to find a suitable route, it increases radius by a factor of First Radius and repeats the above procedure till the iterations $<3$, after which the complete topology is considered for CSPF calculation. If no route CSPF route exists in the whole topology between source and destination node, then that packet is dropped.

\section{Algorithm}

The pseudo-code for the algorithm is as follows:

\section{Algorithm: CASPER}

\section{PARAMETERS}

- N: Total Nodes in the complete Network Topology.

- nodeData: Array which stores information about all nodes in simulation.

- Candidatelist : Array of nodes eligible for CSPF calculation

\section{BEGIN}

Message msg; /* message packet to be transmitted */ If( $\operatorname{src} !=$ NULL \&\& dest != NULL)

\{

Check Route table for available route to destination.

If route satisfies message bandwidth requirements $->$ forward message

Else

\{

radius $=1 / 2$ line(dest, src); //Func to find straight line between src $\&$ dest

$\operatorname{mid}=\operatorname{midpoint}($ dest,src $) ; / /$ Func to find midpoint of line joining src $\&$ dest

first radius = radius;

for $(j=1 ; j<3 \& \&$ !flag $; j++)$

\{

$$
\text { for }(\mathrm{i}=0 ; \mathrm{i}<\mathrm{N} ; \mathrm{i}++)
$$

\{

$\operatorname{if}\left((\text { nodeDate[i].x- mid.x })^{\wedge} 2+(\text { nodeData[i].y- mid.y })^{\wedge} 2<\operatorname{radius}^{\wedge} 2\right)$ candidatelist $[\mathrm{i}]=$ nodeData $[\mathrm{i}]$;

\{

Calculate CSPF distance from this node to other nodes in the area covered by circle;

if ( getroute(dest,src)) flag=1; // function to find route between src \& dest \}

if(!flag)

$\{$ Apply CSPF algorithm on complete topology

if(getroute(dest,src)) Forward Packet;

else Drop Packet; 


\section{Performance Evaluation}

The performance evaluation of the proposed protocol was conducted through simulation analysis using the Qualnet 5.0 simulation package $[13,14,15]$. The simulations were performed by varying the number of nodes and the mobility of the nodes in the network. The various parameters considered for performance evaluation are discussed in Section 5.1 and the simulation results are described in Section 5.2.

\subsection{Performance Metrics}

The performance metrics such as throughput, Packet Delivery Ratio, Average End to End Delay and average jitter that were used for the sake of evaluation of our proposed protocol are discussed in this section.

- Throughput: It is the number of data packets transferred over a period of time. It is usually measured in bits per second.

- Packet Delivery Ratio: It is the ratio of the number of packets received over the number of packets transmitted.

- Average End To End Delay: It is the Average Delay in the transmission of packet from source node to destination node.

- Average Jitter: Packets from the source will reach the destination with different delays due to various factors like network congestion, etc. This variation in delay is known as jitter.

\subsection{Simulation Settings and Results}

The general settings of simulation are provided in Table 1 .

Table 1. General Simulation Settings

\begin{tabular}{l|l}
\hline \multicolumn{1}{c|}{ Name } & \multicolumn{1}{c}{ Value } \\
\hline Terrain Dimensions & $(1500 X 1500) \mathrm{m}^{2}$ \\
\hline Maximum speed & $30 \mathrm{~m} / \mathrm{s}$ \\
\hline Radio Transmission power & $15 \mathrm{~dB}$ \\
\hline CBR Traffic & 2 \\
\hline Number of nodes & 30 \\
\hline Message Bandwidth Requirement & 800 units \\
\hline Node placement & Nodes input \\
\hline Mobility model & Random Waypoint \\
\hline MAC Protocol & IEEE $802.11[16]$ \\
\hline Network Protocol & IPv4 [19] \\
\hline PHY-NOISE-FACTOR & $7.0 \mathrm{~dB}$ \\
\hline PHY-TEMPERATURE & $290 \mathrm{~K}$ \\
\hline Propagation Channel Frequency & $2.4 \mathrm{e} 9 \mathrm{~Hz}$ \\
\hline Propagation Limit & $-111.0 \mathrm{~dB}$ \\
\hline Antenna Gain & 0.0 \\
\hline Antenna Efficiency & 0.8 \\
\hline Antenna Height & $1.5 \mathrm{~m}$ \\
\hline Antenna Model & Omni-directional \\
\hline Link Cost & $500-1000$ units \\
\hline
\end{tabular}


The simulation results are shown with the help of graphs depicted in the next figures.

As can be seen from Figure 4, CASPER performs better than OSPF and OLSR in terms of throughput. This is due to the fact that even with the increased mobility, the packet drops in CASPER are less as it involves congestion reduction mechanism achieved through constrained circular routing. The number of nodes processed in CASPER is also less as most unnecessary nodes get eliminated during the CSPF and circular routing process. In OSPF and OLSR there is no such elimination of unnecessary nodes. OLSR does not perform link database synchronization using a reliable algorithm as done in OSPF and CASPER. Hence, it floods topology data often enough to make sure that the database does not remain unsynchronized for extended periods of time. This may lead to broken links and thus more packet drops.

The average end to end delay for the three protocols versus mobility is shown Figure 5. When compared with each other OLSR gives the best delay whereas OSPF shows worst delay.

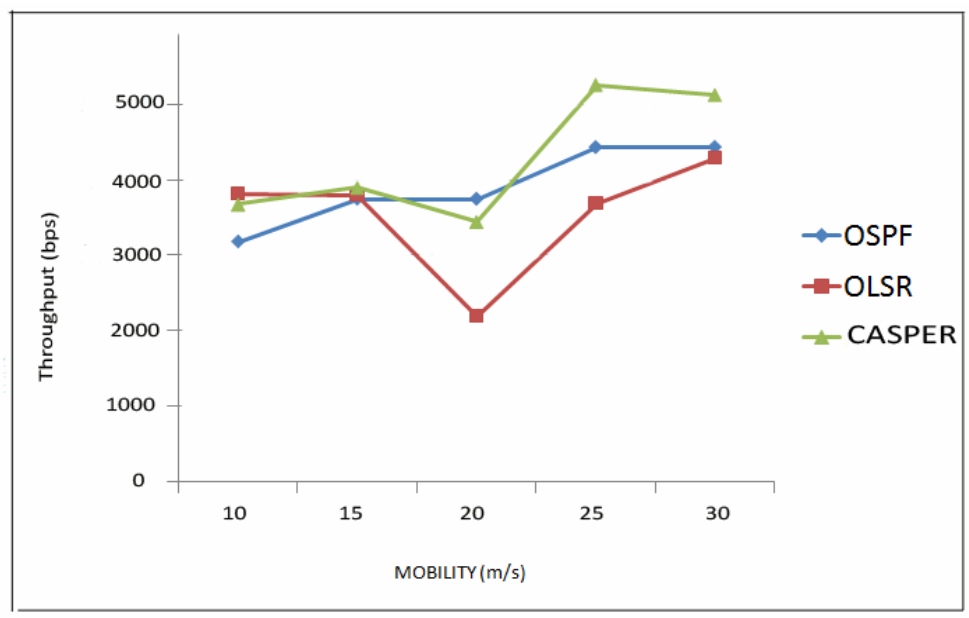

Fig. 4. Throughput Vs. Mobility Of Nodes

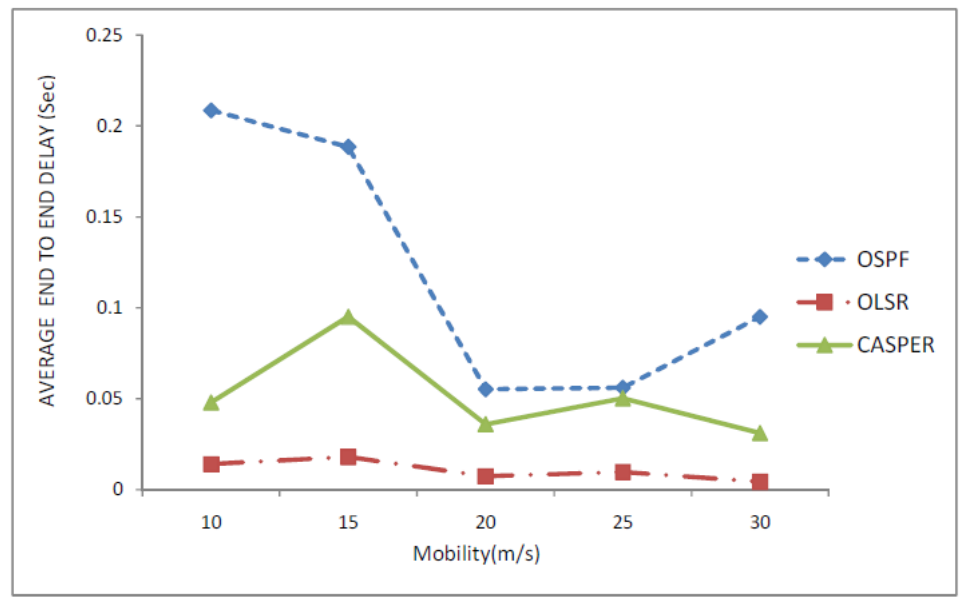

Fig. 5. Average End To End Delay Vs Mobility of Nodes 
CASPER lies between OLSR and OSPF in terms of average end to end delay. OLSR gives best delay due to the fact that here the forwarding path for Topology Control messages is not shared among all nodes. Only a subset of nodes disseminates link state information. Only the links representing MPR selections are advertised. Since here information about only necessary links is maintained the delay in creating routing and neighbor table is less as compared to OSPF where path from a node to all the nodes in the topology is calculated and stored. In CASPER, the path between all the nodes is not calculated. Only those nodes are considered for path calculations, which are probably going to be involved in the routing process. Hence, its delay is less than that of OSPF.

The plots in Figure 6 and Figure 7 show that when the three schemes are compared with each other, it is found that OLSR gives the best jitter. The performance of CASPER lies between OLSR and OSPF. In OLSR information about all the links is not maintained as explained previously, hence the delay in creating routing and neighbor table is less as compared to OSPF where

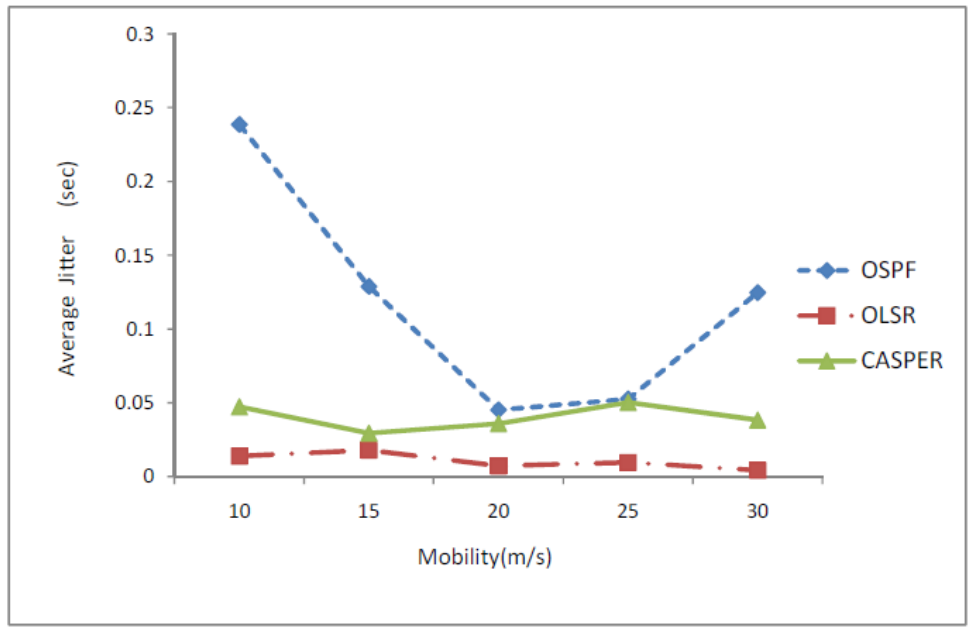

Fig. 6. Average Jitter Vs Mobility Of Nodes

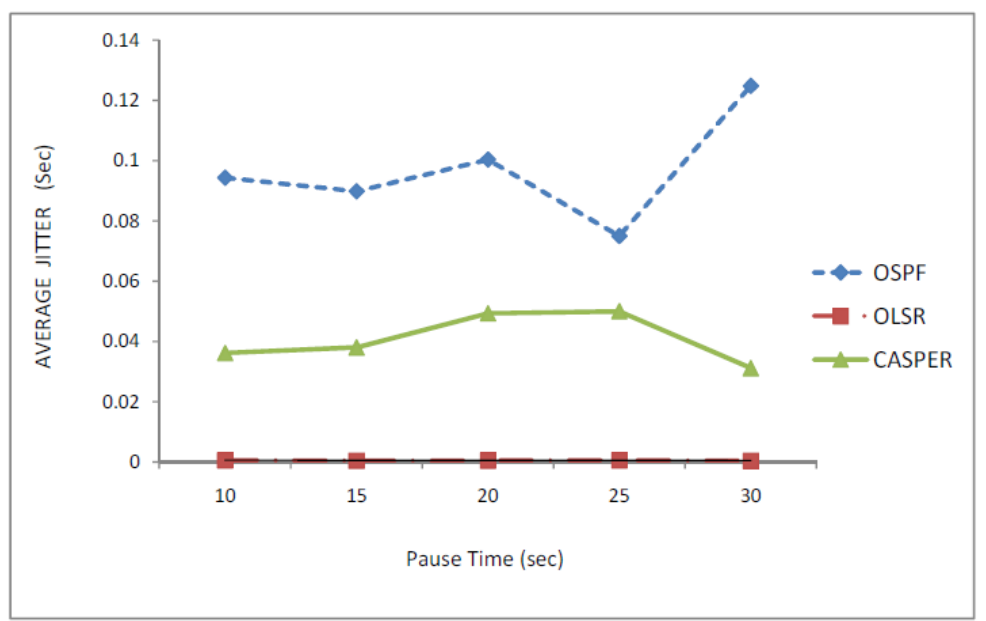

Fig. 7. Average Jitter Vs Pause Time 
all possible links are calculated and stored and then shortest path is calculated. In CASPER not all links are processed but only those which fulfill the constrained circular routing criteria. The average jitter for CASPER remains uniform with increase in node mobility and pause time as clear from the respective figures.

The Packet Delivery Ratio for CASPER is greater than OSPF and OLSR when mobility is varied due to the congestion reduction mechanism applied in CASPER. The number of packet drops is less and packets are transmitted through links that satisfy the minimum criteria specified by constrained circular routing algorithm. OSPF and OLSR contain no such mechanism and hence the packet drops here are more due to congestion in network.

As can be seen from Figure 9, CASPER performs better than OSPF and OLSR in terms of throughput. Also as the pause time is increased the throughput improves. This is due to the fact that the packet drops in CASPER are less as it involves congestion removal mechanism

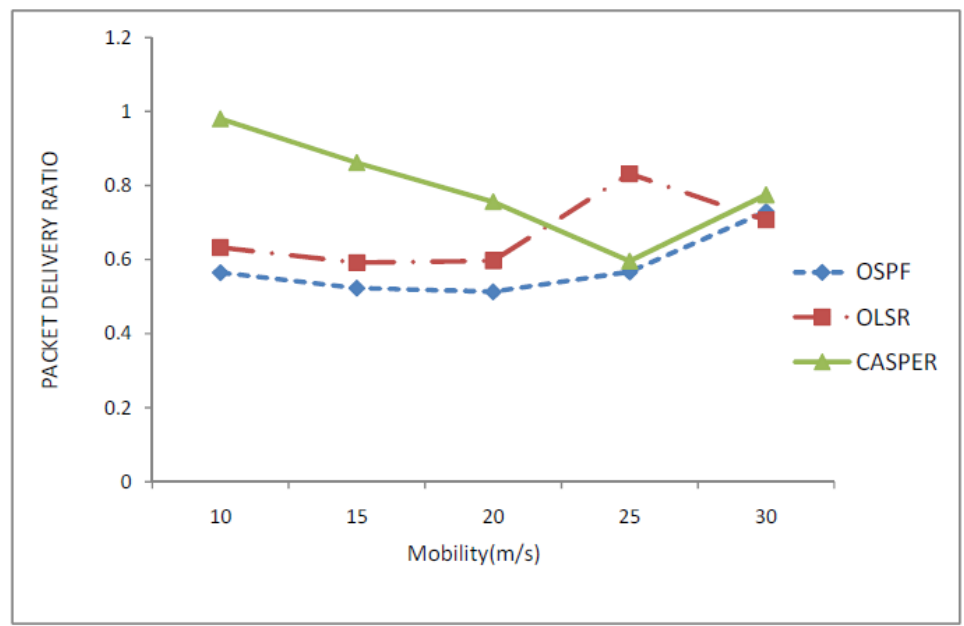

Fig. 8. Packet Delivery Ratio Vs Mobility Of Nodes

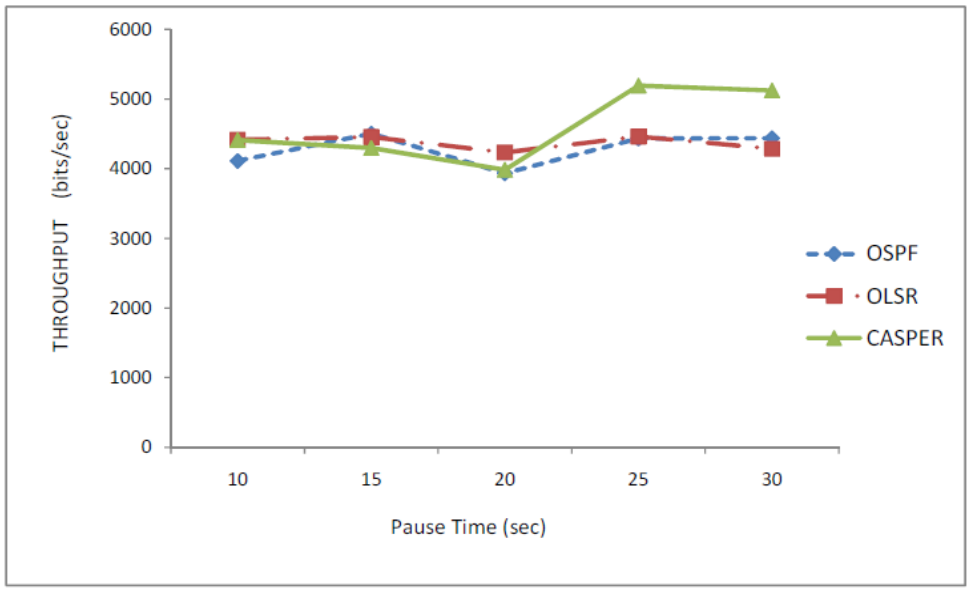

Fig. 9. Throughput Vs Pause Time 
achieved through constrained circular routing. Most unnecessary nodes get eliminated during the CSPF and circular routing process in CASPER. Also link database synchronization is done more frequently in CASPER and OSPF as compared to OLSR, which reduces the probability of dropped packets in CASPER. Hence, the overall the throughput of the network increases. The throughput for CASPER is appreciable at higher pause time as link breaks are less.

Figure 10 depicts the performance of the three protocols with varying pause time. As can be observed, OLSR gives the best delay and OSPF shows worst delay while CASPER lies between OLSR and OSPF. The Delay for OLSR does not change much with increasing pause time. CASPER gives less Delay at higher pause times due to less link breakage at increased pause time. The path selection in CASPER is always better than in OSPF under congested circumstances. Therefore, the performance of CASPER is better than that of OSPF.

Due to efficient routing mechanism and congestion reduction mechanism employed in CASPER its performance in terms of average jitter is better than OSPF as is evident from the Figure 11. Moreover, as the pause time increases the jitter remains almost constant.

As terrain dimensions increase some links get broken as nodes move away from the transmis-

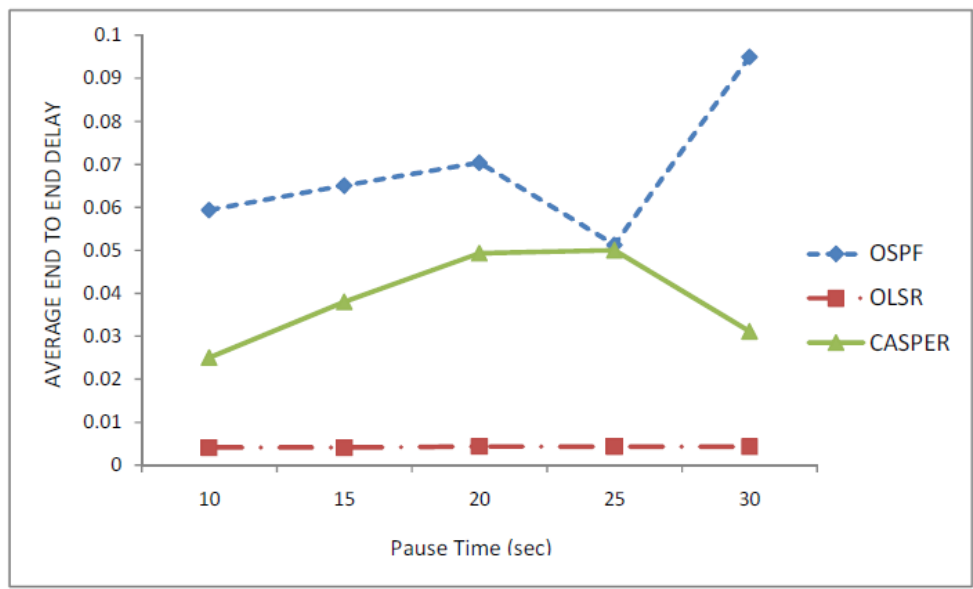

Fig. 10. Average End To End Delay Vs Pause Time

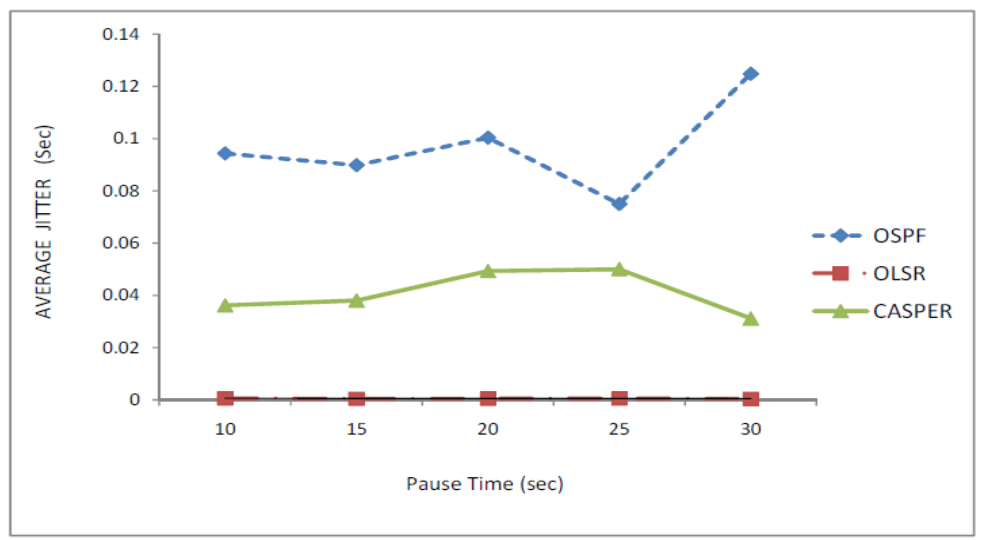

Fig. 11. Average Jitter Vs Pause Time 
sion range of other nodes. This leads to processing of all the nodes in topology and link updating process in OSPF and OLSR. CASPER processes only certain nodes that are included in the circular topology at a time. Therefore, lesser links get updated in the proposed CASPER protocol. Figure 12 shows that CASPER performs better than OSPF with increased terrain dimensions.

As can be seen from Figure 13, CASPER performs better than OSPF and OLSR in terms of throughput. As the terrain dimension is increased the throughput remains almost constant for CASPER. Higher throughput is a result of lesser packet drops in CASPER as it involves congestion removal mechanism achieved through constrained circular routing. As the terrain dimensions increase the distance between the nodes increase and some nodes are removed from the transmission range of other nodes. CASPER performs an efficient check that processes only those nodes that lie within a circle of varying radius. Only those nodes that lie in the transmission range of certain other nodes within the circle are processed. Thus the number of nodes processed in CASPER is less as most unnecessary nodes get eliminated during the CSPF and

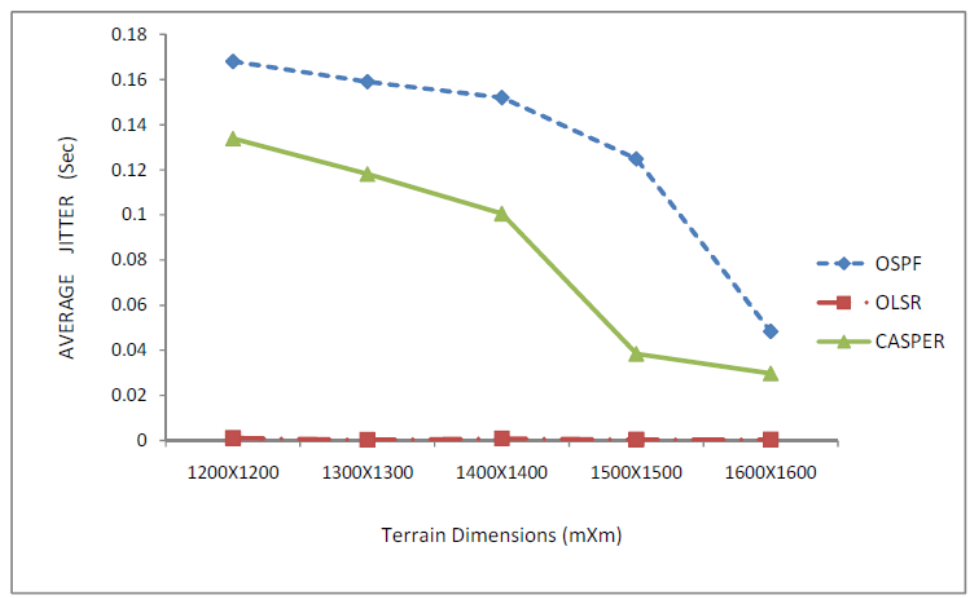

Fig. 12. Average Jitter Vs Terrain Dimensions

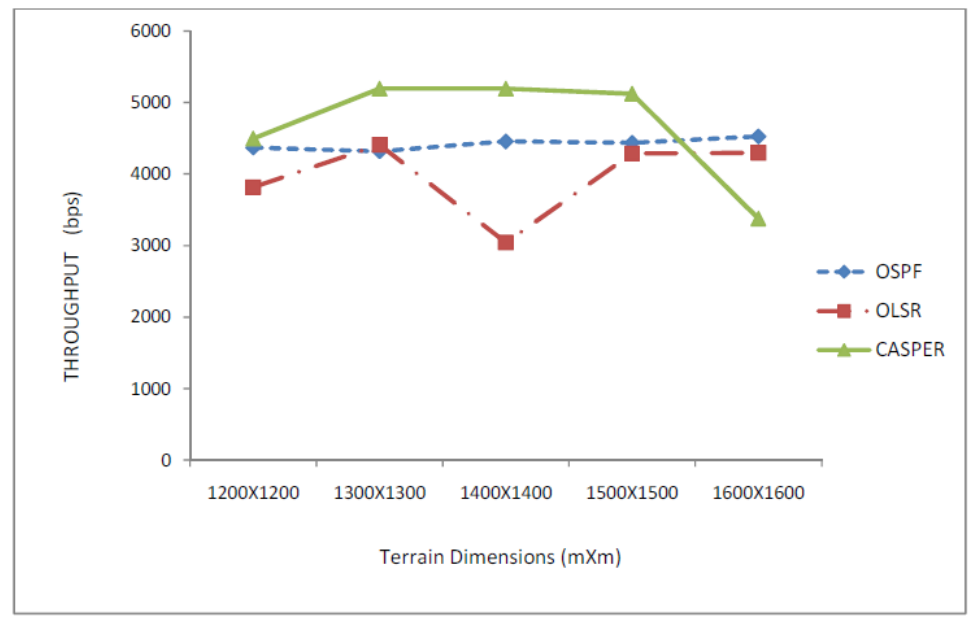

Fia. 13. Throuahput Vs Terrain Dimensions 
circular routing process. In OSPF and OLSR there is no such elimination of unnecessary nodes. With increase in terrain dimensions certain links break and the processing of all the nodes in the topology is restarted. OLSR doesn't perform link database synchronization using a reliable algorithm as done in OSPF and CASPER. So it floods topology data often enough to make sure that the database does not remain unsynchronized for long time. This may lead to broken links and so more packet drops.

As can be observed from Figure 14, the delay in transmission produced in case of CASPER is less than OSPF, but more than OLSR. Both CASPER and OSPF give less jitter with increase in terrain dimensions. OLSR gives constant jitter at all terrain dimensions. CASPER performs better than OSPF due to the application of congestion reduction mechanism and efficient routing of data.

From Figure 15 and Figure 16, it can be observed that the average jitter and end to end delay for CASPER lies between OSPF and OLSR. In CASPER not all links are processed but only

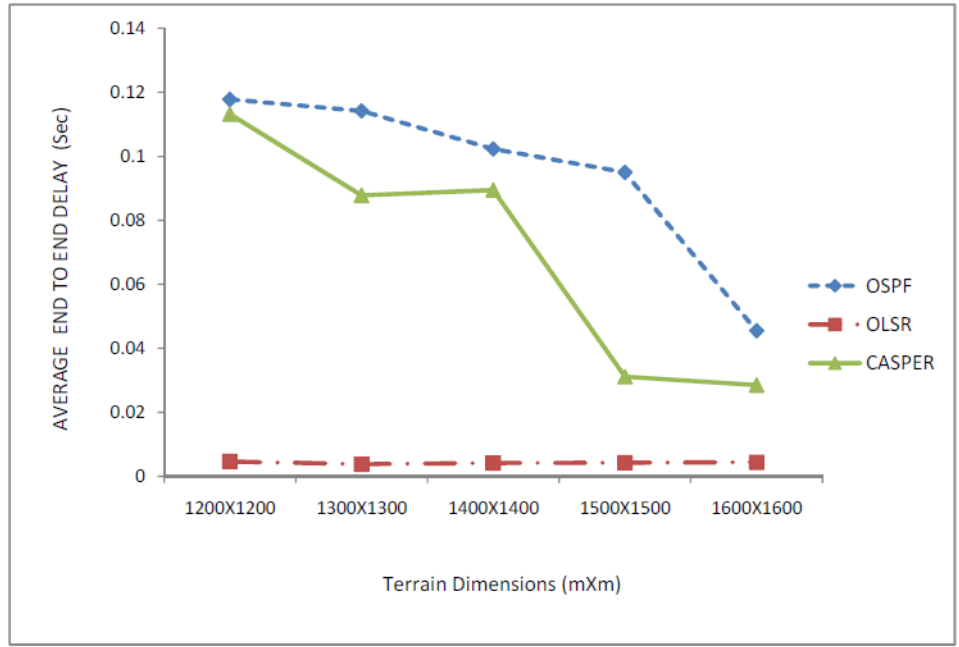

Fig. 14. Average End To End Delay Vs Terrain Dimensions

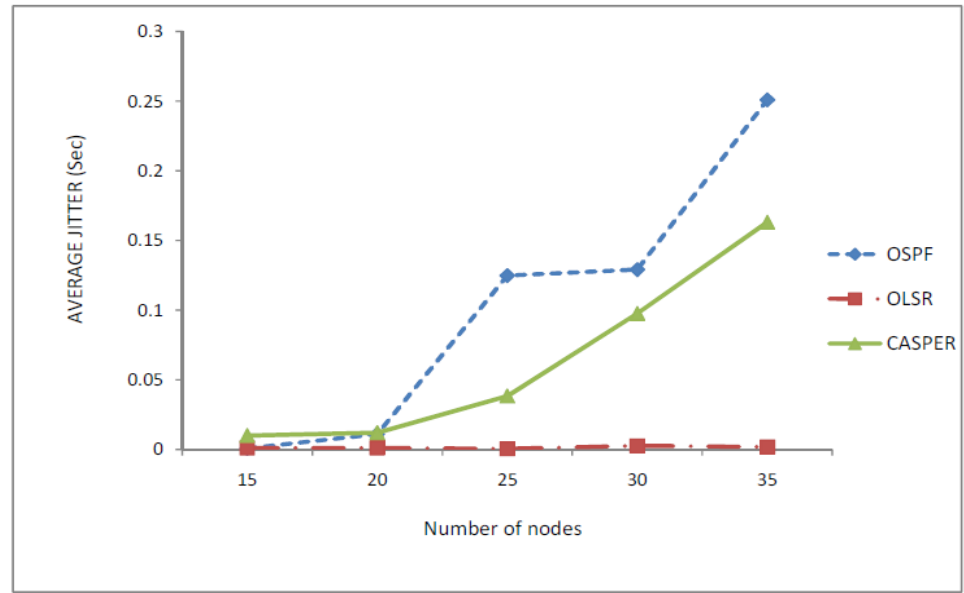

Fig. 15. Average Jitter Vs Number Of Nodes 
those which fulfill the constrained circular routing criteria. As the number of nodes increases, OSPF has to do more calculations to calculate link information between every node. CASPER on the other hand processes only a few certain nodes. Hence, CASPER shows improvement over OSPF. OLSR gives constant jitter and delay with the increasing number of nodes.

As can be seen from Figure 17, CASPER performs better than OSPF and OLSR in terms of throughput. Also as the network becomes denser the throughput improves. This is due to the fact that the packet drops in CASPER are less as it involves congestion removal mechanism achieved through constrained circular routing. The number of nodes processed in CASPER is also less as most unnecessary nodes get eliminated during the CSPF and circular routing process. In OSPF there is no such elimination of unnecessary nodes. OLSR does not perform link database synchronization using a reliable algorithm as done in OSPF and CASPER. Hence, it floods

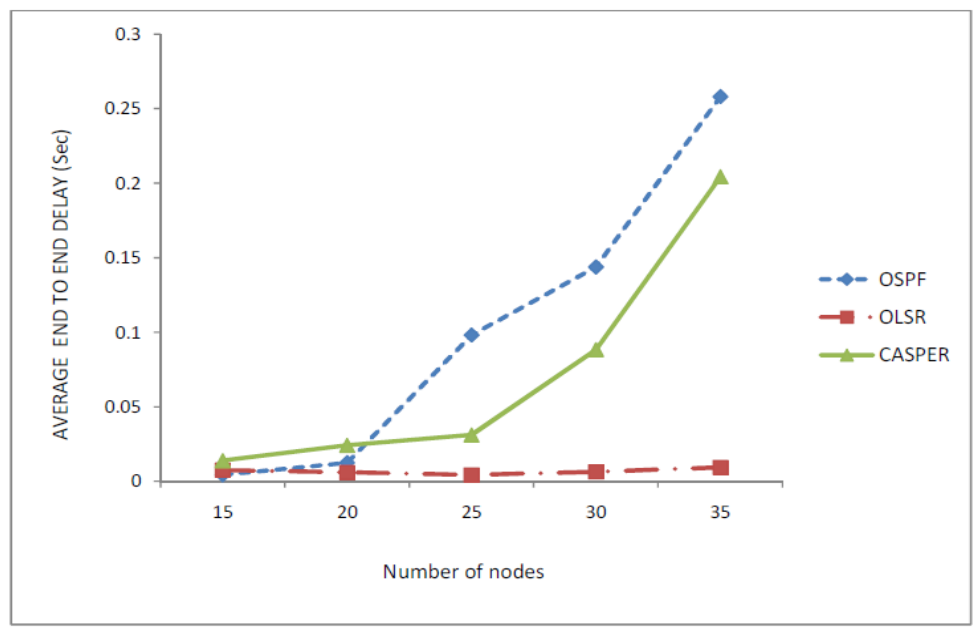

Fig. 16. Average End To End Delay Vs Number Of Nodes

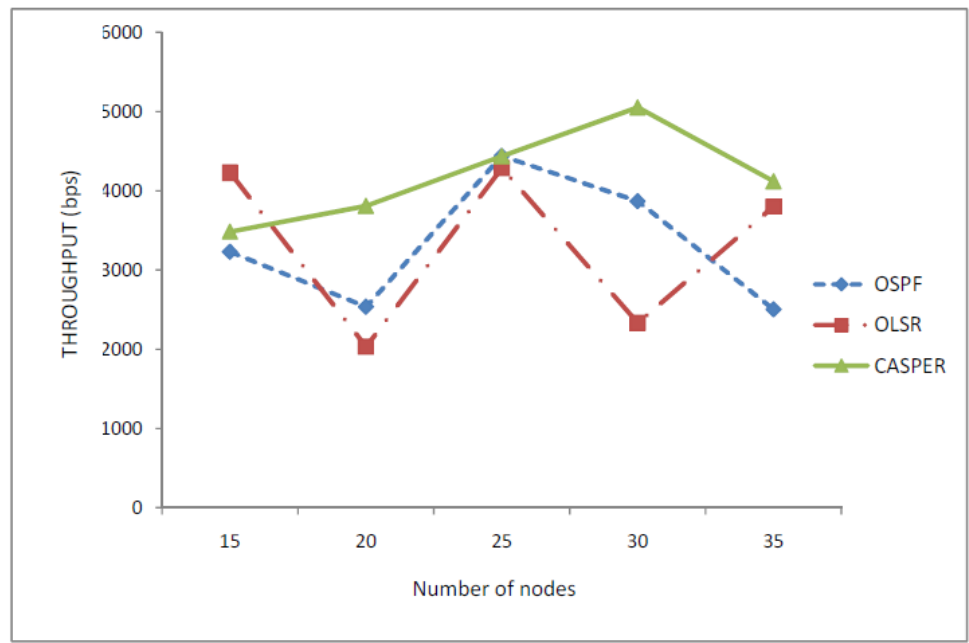

Fig. 17. Throughput Vs Number Of Nodes 
the topology data periodically to make sure that the database does not remain unsynchronized for a long period of time. Else, this may lead to broken links and so more packet drops.

\section{CONCLUSIONS AND FUtURE WORK}

There is a need for congestion free and efficient routing protocols to make Mobile Ad Hoc Networks (MANETs) more useful in transmitting data with high bandwidth requirement. The CASPER protocol takes care of the bandwidth requirement of data while transmitting it. It not only minimizes delay, it also maximizes the throughput in a network during routing. It decreases the average drop in packets and shows an improvement in terms of reduction of traffic congestion by taking care of the bandwidth requirements of the data to be transmitted. Moreover, the unnecessary calculations and processing of each node is avoided, thereby saving time. It is also space efficient as it involves less storage consumption in routing tables because only the constrained shortest paths from a source to the destination node and intermediate nodes are stored at a time instead of paths between all possible nodes in topology, which are not even involved in routing. OSPF and OLSR store paths between all nodes before beginning the routing process. All these are the improvements of CASPER over OSPF. CASPER has also been compared with OLSR and shows improvements in terms of throughput and packet delivery ratio during routing of bulky data.

CASPER has been compared with OSPF and OLSR because it is a link state MANET protocol just as these are and it uses OSPF as its base protocol. In the future, we plan to compare it with other congestion aware MANET protocols. Currently, the protocol works by considering the whole topological area as one autonomous system. In future it can be implemented for areas divided into more than one autonomous system; each having its own designated router. Furthermore, the incremental SPF technique designed by CISCO [18] can be incorporated to make CASPER even more efficient under congested scenarios.

\section{REFERENCES}

[1] S. Corson, J. Macker, RFC2501 - Mobile Ad hoc Networking (MANET): Routing Protocol, January 1999.

[2] Internet Engineering Task Force, "Manet working group charter", http://www.ietf.org/html.charters/ manet-charter.html

[3] C-2328 OSPF v2. 1997. W. V. Wollman and Y. Barsoum. "Overview of Open Shortest Path First, version 2 (ospf v2) routing in the tactical environment”, Proc. of IEEE MILCOM., Vol. 3, pp. 925930, October 1995.

[4] Moy J, RFC-2328 OSPF v2. 1997.

[5] P. Jacquet, P. Muhlethaler, A. Qayyum, A Laouiti,L. Viennot, T Clausen, MANET draft “draft-ietfmanet-olsr-02.txt",2000.

[6] T. Clausen, P. Jacquet, Project Hipercom, INRIA, RFC-3626: Optimized Link State Routing Protocol (OLSR), October 2003.

[7] T. H. Clausen, G. Hansen, L. Christensen, G. Behrmann, "The Optimized Link State Routing Protocol Evaluation through Experiments and Simulation”, in Proc. of Wireless Personal Mobile Communications, Aalborg, Denmark, 2001.

[8] Anis Laouti, Paul M"uhlethaler, Abdellah Najid, Epiphane Plakoo. "Simulation Results of the OLSR Routing Protocol for Wireless Networks". 1st Mediterranean Ad-Hoc Networks workshop (Med-HocNet), 2002.

[9] Satish Jamadagni, Praveen C H, "OSPF extensions for flexible CSPF algorithm support", Draftsatish-ospf-cspf-support-00, October 2002. 
[10] A. Qayyum, Laurent Viennot, Anis Laouiti. "Multipoint Relaying for flooding broadcast messages in mobile wireless networks” Proc. 35th Ann. Hawaii Int'l Conf. System Sciences (HICSS-35), IEEE CS Press, pp. 298-307, 2002.

[11] P. Jacquet, P. Minet, P. Muhlethaler, N. Rivierre,'Increasing reliability in cable free radio LANs: Low level forwarding in HIPERLAN", Wireless Personal Communications, Vol 4, No 1, pp. 51-63, 1997.

[12] Y. Yong and M. Lingjuan, "GDOP results in all-in-view positioning and in four optimum satellites positioning with GPS PRN codes ranging", Position Location and Navigation Symposium, 2004 (PLANS 2004), pp. 723-727, 2004.

[13] Qualnet 4.5 Programmer's Guide, 2008 http://www.eurecom.fr/ chenj/QualNet03.pdf.

[14] Qualnet, http://www.scalablenetwork.com/products/qualnet/.

[15] QualNet 4.5 Multimedia and Enterprise Model Library, March 2008.

[16] “Wireless LAN Medium Access Control (MAC) and Physical Layer (PHY) Specifications”, IEEE 802.11 Standard, 1997.

[17] http://en.wikipedia.org/wiki/File:CSPF-Network.JPG

[18] http://www.cisco.com/en/US/docs/ios/12_0s/feature/guide/ospfispf.pdf

[19] RFC 791 Internet Protocol, DARPA Internet Program Protocol Specification, September 1981.

[20] E. Baccelli, D. Nguyen ,CRC, T. Clausen Lix. "OSPF Multipoint Relay (MPR) Extension for Ad Hoc Networks". Draft-ietf-ospf-manet-mpr-04.

[21] Richard Ogier. Presented by Tom Henderson. "OSPF-MDR Position". Draft-ogier-ospf-mdr-position-00.txt.

[22] D. Katz, K. Kompella, D. Yeung. “Traffic Engineering (TE) Extensions to OSPF Version 2". Draftkatz-yeung-ospf-traffic-01.

[23 ]J.-L. Le Roux, J.-P. Vasseur, J. Boyle., "Requirements for Inter-Area MPLS Traffic Engineering", $R F C$ 4105, June 2005.

[24] M. S. Obaidat, S. K. Dhurandher, D. Gupta, N. Gupta and A. Asthana, "DEESR: Dynamic Energy Efficient and Secure Routing Protocol for Wireless Sensor Networks in Urban Environments", Journal of Information Processing Systems, Vol. 6, No. 3, pp. 269-294, KIPS, 2010.

\section{Mohammad S. Obaidat}

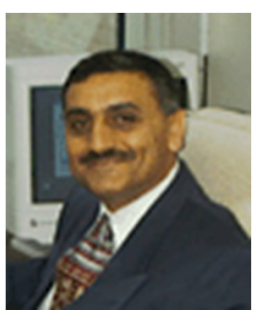

Professor Mohammad S. Obaidat (Fellow of IEEE and Fellow of SCS) is an internationally well known academic/researcher/ scientist. He received his Ph.D. and M. S. degrees in Computer Engineering with a minor in Computer Science from The Ohio State University, Columbus, Ohio, USA. Dr. Obaidat is currently a full Professor of Computer Science at Monmouth University, NJ, USA. Among his previous positions are Chair of the Department of Computer Science and Director of the Graduate Program at Monmouth University and a faculty member at the City University of New York. He has received extensive research funding and has published over Ten (10) books and over Four Hundred and seventy fie (475) refereed technical articles in scholarly international journals and proceedings of international conferences, and currently working on three more books. Prof. Obaidat is the author of a new upcoming book: Wireless Sensor Networks (Cambridge University Press). He is also the editor to 2 new upcoming books: Cooperative Networking (John Wiley \&Sons 2010) and Pervasive Computing and Networking (John Wiley \&Sons 2010). Prof. Obaidat is the author of the book entitled: "Fundamentals of Performance Evaluation of Computer and Telecommunications Systems," by John Wiley \& Sons in 2010. Dr. Obaidat is the Editor of the Book entitled, "E-business and Telecommunication Networks", published by Springer in 2008. He is the co-author of the book entitled, "Security of e-Systems and Computer Networks" published by Cambridge University Press in 2007. He is the co-author of the Best Selling Book, "Wireless Networks" and "Multiwavelength Optical LANs" published by John Wily \& Sons (2003). Obaidat is the 
editor of the book, APPLIED SYSTEM SIMULATION: Methodologies and Applications, published by Kluwer (now Springer) in 2003. Professor Obaidat has served as a consultant for several corporations and organizations worldwide. Mohammad is the Editor-in-Chief of the Wiley International Journal of Communication Systems, the FTRA Journal of Convergence and the KSIP Journal of Information Processing. He served as an Editor of IEEE Wireless Communications from 2007-2010. Between 1991-2006, he served as a Technical Editor and an Area Editor of Simulation: Transactions of the Society for Modeling and Simulations (SCS) International, TSCS. He also served on the Editorial Advisory Board of Simulation. He is now an editor of the Wiley Security and Communication Networks Journal, Journal of Networks, International Journal of Information Technology, Communications and Convergence, IJITCC, Inderscience. He served on the International Advisory Board of the International Journal of Wireless Networks and Broadband Technologies, IGI-global. Prod. Obaidat is an associate editor/ editorial board member of seven other refereed scholarly journals including two IEEE Transactions, Elsevier Computer Communications Journal, Kluwer Journal of Supercomputing, SCS Journal of Defense Modeling and Simulation, Elsevier Journal of Computers and EE, International Journal of Communication Networks and Distributed Systems, The Academy Journal of Communications, International Journal of BioSciences and Technology and International Journal of Information Technology. He has guest edited numerous special issues of scholarly journals such as IEEE Transactions on Systems, Man and Cybernetics, SMC, IEEE Wireless Communications, IEEE Systems Journal, SIMULATION: Transactions of SCS, Elsevier Computer Communications Journal, Journal of C \& EE, Wiley Security and Communication Networks, Journal of Networks, and International Journal of Communication Systems, among others. Obaidat has served as the steering committee chair, advisory Committee Chair and program chair of numerous international conferences including the IEEE Int'l Conference on Electronics, Circuits and Systems, IEEE International Phoenix Conference on Computers and Communications, IEEE Int'l Performance, Computing and Communications Conference, IEEE International Conference on Computer Communications and Networks, SCS Summer Computer Simulation Conference, SCSC'97, SCSC98-SCSC2005, SCSC2006, the International Symposium on Performance Evaluation of Computer and Telecommunication Systems since its inception in 1998, International Conference on Parallel Processing, Honorary General Chair of the 2006 IEEE Intl. Joint Conference on E-Business and Telecommunications, ICETE2006. He served as General Co-Chair of ICETE 2007-ICETE 2010. He has served as the Program Chair of the International Conference on Wireless Information Networks and Systems from 2008-Presnet. He is the co-founder and Program Co-Chair of the International Conference on Data Communication Networking, DCNET since its inception in 2009. Obaidat has served as the General Chair of the 2007 IEEE International Conference on Computer Systems and Applications, AICCSA2007, the IEEE AICCSA 2009 Conference. and the 2006 International Symposium on Ad hoc and Ubiquitous Computing (ISAHUC'06). He is the founder of the International Symposium on Performance Evaluation of Computer and Telecommunication Systems, SPECTS and has served as the General Chair of SPECTS since its inception. Obaidat has received a recognition certificate from IEEE. Between 1994-1997, Obaidat has served as distinguished speaker/visitor of IEEE Computer Society. Since 1995 he has been serving as an ACM distinguished Lecturer. He is also an SCS distinguished Lecturer. Between 1996-1999, Dr. Obaidat served as an IEEE/ACM program evaluator of the Computing Sciences Accreditation Board/Commission, CSAB/CSAC. Obaidat is the founder and first Chairman of SCS Technical Chapter (Committee) on PECTS (Performance Evaluation of Computer and Telecommunication Systems). He has served as the Scientific Advisor for the World Bank/UN Digital Inclusion Workshop- The Role of Information and Communication Technology in Development. Between 1995-2002, he has served as a member of the board of directors of the So- 
ciety for Computer Simulation International. Between 2002-2004, he has served as Vice President of Conferences of the Society for Modeling and Simulation International SCS. Between 2004-2006, Prof. Obaidat has served as Vice President of Membership of the Society for Modeling and Simulation International SCS. Between 2006-2009, he has served as the Senior Vice President of SCS. Currently, he is the President of SCS. One of his recent co-authored papers has received the best paper award in the IEEE AICCSA 2009 international conference. He also received the best paper award for one of his papers accepted in IEEE GLOBCOM 2009 conference. Dr. Obaidat received very recently the Society for Modeling and Simulation Intentional (SCS) prestigious McLeod Founder's Award in recognition of his outstanding technical and professional contributions to modeling and simulation. He received in Dec 2010, the IEEE ComSoc- GLOBECOM 2010 Outstanding Leadership Award for his outstanding leadership of Communication Software Services and Multimedia Applications Symposium, CSSMA 2010. He has been invited to lecture and give keynote speeches worldwide. His research interests are: wireless communications and networks, telecommunications and Networking systems, security of network, information and computer systems, security of e-based systems, performance evaluation of computer systems, algorithms and networks, high performance and parallel computing/computers, applied neural networks and pattern recognition, adaptive learning and speech processing. Recently, Prof. Obaidat has been awarded a Nokia Research Fellowship and the distinguished Fulbright Scholar Award. During the 2004/2005, he was on sabbatical leave as Fulbright Distinguished Professor and Advisor to the President of Philadelphia University in Jordan, Dr. Adnan Badran. The latter became the Prime Minister of Jordan in April 2005 and served earlier as Vice President of UNESCO. Prof. Obaidat is a Fellow of the Society for Modeling and Simulation International SCS, and a Fellow of the Institute of Electrical and Electronics Engineers (IEEE). For more info; se: http://bluehawk.monmouth.edu/mobaidat/.

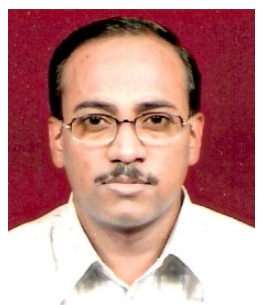

\section{Sanjay Kumar Dhurandher}

He received the M. Tech. and Ph.D. Degrees in Computer Sciences from the Jawaharlal Nehru University, New Delhi, India.

$\mathrm{He}$ is presently working as an Associate Professor in the Division of Information Technology, Netaji Subhas Institute of Technology (NSIT), University of Delhi, India. He is also the Head of the Advanced Centre CAITFS, Division of Information Technology, NSIT, University of Delhi. From 1995 to 2000 he worked as a Scientist/Engineer at the Institute for Plasma Research, Gujarat, India which is under the Department of Atomic Energy, India. His current research interests include wireless ad-hoc networks, sensor networks, computer networks, network security and Underwater Sensor Networks. He is serving as the Associate Editor of Wiley's International Journal of Communication Systems.

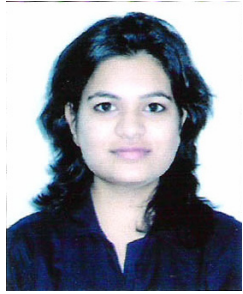

\section{Khushboo Diwakar}

She is currently a Java Developer at Oracle and works on the Planning and Optimization of Retail Products using the Application Development Framework of Oracle. She graduated from the Netjai Subhas Institute of Technology in Information Technology, University of Delhi in year 2010 and has a strong interest in designing of ad hoc network routing protocols. 